\title{
Interfacial adsorption in two-dimensional pure and random-bond Potts models
}

\author{
Nikolaos G. Fytas ${ }^{1}$, Panagiotis E. Theodorakis ${ }^{2}$, and Anastasios Malakis ${ }^{1,3}$ \\ ${ }^{1}$ Applied Mathematics Research Centre, Coventry University, Coventry CV1 5FB, United Kingdom \\ ${ }^{2}$ Institute of Physics, Polish Academy of Sciences, \\ Al. Lotników 32/46, 02-668, Warsaw, Poland and \\ ${ }^{3}$ Department of Physics, Section of Solid State Physics, \\ University of Athens, Panepistimiopolis, GR 15784 Zografou, Greece
}

(Dated: February 23, 2018)

\begin{abstract}
We study using Monte Carlo simulations the finite-size scaling behavior of the interfacial adsorption of the two-dimensional square-lattice $q$-states Potts model. We consider the pure and random-bond versions of the Potts model for $q=3,4,5,8$ and $q=10$, thus probing the interfacial properties at the originally continuous, weak, and strong first-order phase transitions. For the pure systems our results support the early scaling predictions for the size dependence of the interfacial adsorption at both first- and second-order phase transitions. For the disordered systems, the interfacial adsorption at the (disordered induced) continuous transitions is discussed, applying standard scaling arguments and invoking findings for bulk critical properties. The self-averaging properties of the interfacial adsorption are also analyzed by studying the infinite limit-size extrapolation of properly defined signal-to-noise ratios.

PACS numbers: $05.50 .+\mathrm{q}, 75.10 . \mathrm{Hk}, 75.10 . \mathrm{Nr}$
\end{abstract}

\section{INTRODUCTION}

Critical interfacial phenomena have been studied extensively over the last decades, both experimentally and theoretically [1 [4]. A well-known example is wetting, where the macroscopically thick phase, e.g., the fluid, is formed between the substrate and the other phase, say, the gas. Liquid and gas are separated by the interface. An interesting complication arises when one considers the possibility of more than two phases. A third phase may be formed at the interface between the two other phases. An experimental realization is the two-component fluid system in equilibrium with its vapor phase [2, 5]. Both of the above scenarios may be mimicked in statistical physics in a simplified fashion, by either the two-state Ising model in wetting - with the state " +1 " representing, say, the fluid, and "- 1 " the gas - or for the case of a third phase via multi-state spin models, simply by fixing distinct boundary states at the opposite sides of the system. In this latter case, the formation of the third phase with an excess of the non-boundary states has been called as interfacial adsorption [6, 7].

Throughout the years, various aspects of the interfacial adsorption have been investigated via Monte Carlo methods and density renormalization-group calculations on the basis of specific multi-state spin models, namely Potts and Blume-Capel models [6, 8-16]. Additional scaling and analytic arguments have been presented [,, 9 , 12 , 17 20], though not all of them have been concretely confirmed numerically, due to the restricted system sizes studied and the apparent underlying scaling corrections (in some cases also because of the uncertainties in the location of critical points). However, notable results in the field include the determination of critical exponents and scaling properties of the temperature and lattice-size dependencies, as well as the clarification of the fundamental role of the type of the bulk transition, with isotropic scaling holding at continuous and tricritical bulk transitions, and anisotropic scaling at bulk transitions of first-order type.

More recently, the role of randomness on the interfacial properties has been studied [21] and was found to affect, especially, the position of the interface, the excess or interfacial adsorption, and the form of the histograms resulting from the different random realizations. Still, predictions of the isotropic finite-size scaling description for the interfacial adsorption at continuous phase transitions were observed to hold, at least for the particular case of the dilute 8-states Potts model studied in Ref. 21]. Attention should be drawn to related previous work on interfacial phenomena in dilute ferromagnetic Potts models, in particular, considering hierarchical lattices, i.e., applying the Migdal-Kadanoff real space renormalization to the square lattice [22], or performing a preliminary Monte Carlo study for the square lattice model [23].

Motivated by Ref. [21], in the present work we study the scaling behavior of the interfacial adsorption of several two-dimensional pure and random-bond Potts models. In particular we consider the disordered $q=3$ and pure $q=8$ models, that complement our previous work 21], and we furthermore extend these studies by presenting new results for both the pure and disordered versions of the $q=4,5$, and $q=10$ models. For the case of pure and randomness-induced continuous transitions we present concrete numerical evidence in favor of the standard isotropic scaling with exponents that can be traced back to the best-known estimates of the bulk critical exponent ratio $\beta / \nu$ of the Potts model, where $\beta$ and $\nu$ are the bulk critical exponents of the order parameter and correlation length, respectively, thus reinforcing the main result of Ref. 21] for the $q=8$ case. For the first- 
order phase transitions corresponding to the pure $q=5$, 8 , and $q=10$ Potts models, our numerical data and scaling analysis strongly support the early scaling predictions for the size dependence of the interfacial adsorption at first-order transitions [9]. In the present paper we also discuss the self-averaging properties of the interfacial adsorption of the disordered Potts models in terms of properly defined signal-to-noise ratios, an aspect that hasn't been considered before in the literature. Various forms of corrections-to-scaling are discussed and depending on the number of states $q$ of the Potts model some expectations from the literature are used as the best possible choices. However, the overall observed scaling behavior does not drastically change by the use of such corrections.

The outline of the article is as follows: In Sec. II the model and the interfacial adsorption are introduced and in Sec. III the numerical method implemented is outlined. Our main finite-size scaling analysis and results are presented in Sec. IV. The summary, Sec. V] concludes the article.

\section{MODEL AND INTERFACIAL ADSORPTION}

We study the nearest-neighbor $q$-states Potts model on the square lattice described by the Hamiltonian

$$
\mathcal{H}=-\sum_{\langle i j\rangle} J_{i j} \delta_{\sigma_{i}, \sigma_{j}}
$$

The Potts variable at site $i, \sigma_{i}$, takes the values $1,2, \ldots, q$ [24] and the ferromagnetic random couplings $J_{i j}>0$ between nearest-neighbor sites $i$ and $j$ are either $J_{1}$, with probability $p$, or $J_{2}$, with probability $1-p$. In the case $J_{1}>J_{2}$, one has either strong or weak bonds. Then, the ratio $r=J_{2} / J_{1}$ defines the disorder strength. Clearly, the value $r=1$ corresponds to the pure model.

In this article, we shall consider the system at its selfdual point, where both couplings occur with the same probability, $p=1 / 2$. Then, the phase-transition temperatures between the ordered ferromagnetic phase and the high-temperature disordered phase are known exactly from self-duality for arbitrary values of the internal states $q$ and disorder-strength ratios $r$ [25]

$$
\left(e^{\left(J_{1} / k_{\mathrm{B}} T_{\mathrm{c}}\right)}-1\right)\left(e^{\left(r J_{1} / k_{\mathrm{B}} T_{\mathrm{c}}\right)}-1\right)=q .
$$

From the above equation one may easily numerically calculate $k_{\mathrm{B}} T_{\mathrm{c}} / J_{1}$ for any given value of $r(r=1 / 10$ at the present study). Thus, via Eq. (2), analyzes on the critical behavior of the interfacial adsorption of the disordered Potts model, based on Monte Carlo simulation data, as it is also done in the present paper, are significantly simplified.

Bulk criticality of such disordered Potts models on the square lattice has attracted much interest, partly, because the transition is of continuous type for all values of $q$, while being, in the pure case, of first order for
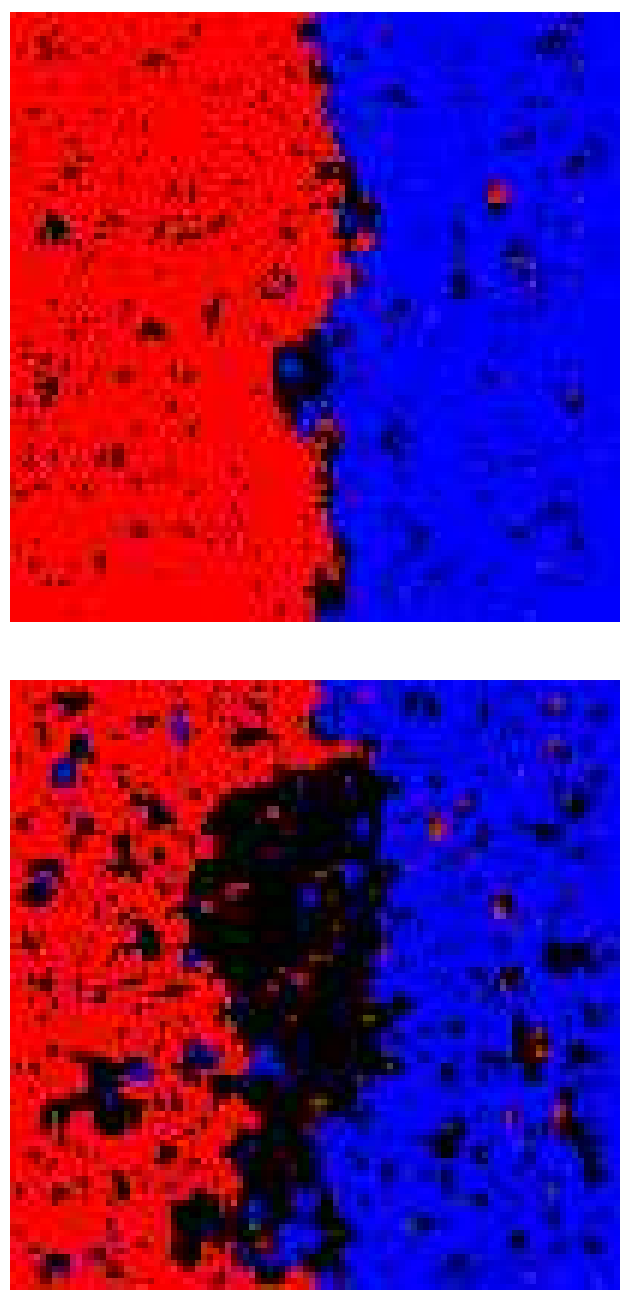

FIG. 1: (color online) Typical equilibrium Monte Carlo configurations of an $L=100, q=10$, pure (upper panel) and random-bond $r=1 / 10$ (lower panel) Potts model at $k_{\mathrm{B}} T / J_{1}=0.98 k_{\mathrm{B}} T_{\mathrm{c}} / J_{1}$. In both cases, red color depicts the $q=1$ states, blue color the $q=2$ states, whereas the nonboundary states $(q \geq 3)$ adsorbed at the interface are shown blackened. Note that the fixed boundaries $[1: 2]$ are also included in these illustrations.

$q>4$ [24, 26]. Exact values of the critical exponents are only known in the clean case [24]. Numerical analysis, in the dilute case suggest that the bulk critical exponents depend rather mildly on $q[26]$. Then, the analysis of the interfacial adsorption in these models may be simplified by the fact, that isotropic finite-size scaling is expected to hold at continuous transitions [6, 9, 10]. Static and dynamic bulk critical properties of the disordered Potts models have been estimated, using a variety of, predominantly, numerical methods [26].

The degeneracy between the $q$ equivalent Potts states may be lifted by appropriate boundary conditions. In order to study the interfacial adsorption, denoted hereafter as $W$, we shall employ special boundary conditions, dis- 
tinguishing the cases $[1: 1]$ and $[1: 2]$. For the case $[1: 1]$, the Potts variable is set, at all boundary sites, equal to $q=1$, while for the case $[1: 2]$, the variable is set equal to 1 at one half of the boundary sites, and to 2 at the opposite half of the boundary sites. Then, the boundary condition [1:2] introduces an interface between the 1rich domain (or phase) and the 2-rich domain (or phase). By examining typical Monte Carlo equilibrium configurations, as shown in Fig. 1 for an instance of the pure (upper panel) and disordered (lower panel) $q=10$ Potts model, it is seen that at the interface between the 1- and and 2-rich domains an excess of the non-boundary states is generated compared to the case of the absence of an interface. As expected, in the dilute case, the position of the interface, as well as the extent of the intervening third phase of non-boundary states, may be strongly affected by the spatial distribution of the couplings.

Then, the interfacial adsorption measuring the surplus of non-boundary states induced by the interface between the 1- and 2-rich regions for lattices with $L^{2}$ nonboundary sites, where $L$ denotes the linear dimension of the lattice, is defined following Eq. (2) of Ref. [12] by

$$
W=\frac{1}{L} \sum_{n} \sum_{i}\left(\left\langle\delta_{\sigma_{i}, n}\right\rangle_{[1: 2]}-\left\langle\delta_{\sigma_{i}, n}\right\rangle_{[1: 1]}\right) .
$$

The summation is over all non-boundary sites $i$ and all non-boundary states $n=3,4, \ldots, q$ and the thermal average is taken. For a disordered system now, the above definition denotes the interfacial adsorption of a single realization. In this case, a second average over the disorder distribution needs to be taken, as it is also done in the present work. The above definition of the interfacial adsorption has been successfully used in the past [6, $, \underline{8}, 12]$ and although its implementation demands Monte Carlo simulations on two systems with different boundary conditions, it offers a simple and physically appealing method to estimate the width of the interface by reflecting quantitatively the difference in fluctuations of the two systems. Thus, $W$ is geometrically interpreted as the effective width of the domain of non-boundary states between the 1- and 2-rich domains. In the case of secondorder phase transitions, normalizing Eq. (3) with $1 / L$ produces an effective width that gives divergencies of the form $\sim L^{(1-\beta / \nu)}[6,[8,9]$. However, one may also argue that it makes more sense to normalize Eq. (3) with $1 / L^{2}$. In this case the corresponding effective width scales as $\sim L^{-\beta / \nu}$ with the system size and we should recognize that in this practise the effective width becomes of zero measure compared to the system's size.

\section{SIMULATION DETAILS}

In our simulations of the Potts models we applied the Metropolis [27] and the cluster-flip Wolff algorithm [28]. Of course, cluster flips violating the boundary conditions are not allowed [29]. As usual, small lattices may be simulated using the Metropolis algorithm, while the Wolff algorithm [28] is more efficient and is preferred for larger, say $L>30$, system sizes [30]. Overall, we studied lattices with up to $100 \times 100$ sites for both pure and disordered Potts models. Only for the pure $q=3$ Potts model data for system sizes up to $200^{2}$ sites have been generated and taken from Ref. [21].

Certainly, equilibration and averaging times depend on the lattice size. Moreover, for disordered models, we observed that the given bond realization may affect these times. In the case of the Metropolis algorithm, eventually, simulations with $10^{7}$ Monte Carlo steps per site for $L=10$ were performed, increasing the length of the runs, roughly, with $L^{2}$. On the other hand, for the application of the Wolff algorithm, the number of (Wolff) clusters used in our simulations varied from $2 \times 10^{7}$ for the smaller systems sizes up to $3 \times 10^{9}$ for the larger sizes considered. The Wolff clusters are constructed as usual with the appropriate acceptance probability from the set of the neighboring lattice sites sharing the same value of the spin [28].

In disordered systems, the main source of errors stems from the fact that the simulation data may vary drastically among different random configurations. In this work, the corresponding histograms or distributions have been recorded for $r=1 / 10$ and all values of $q$ considered. Noteworthy, bulk properties of the random-bond Potts model for various values of $r$ and $q$ have been studied quite extensively before [26, 31 37] and the obtained pool of results will prove to be extremely useful for the analysis in the following Section. The histograms at the critical point show nearly Gaussian shapes, but being weakly tailed, in accordance with previous observations and discussions in our previous work [21] and in Ref. 22] for the dilute Potts models on hierarchical lattices. The standard errors resulting from an ensemble average over bond realizations decrease with the number of configurations, $\mathcal{N}$, as $\sim 1 / \sqrt{\mathcal{N}}$. The proportionality factor seems to become somewhat smaller for larger lattices. To obtain reasonable accuracy, we averaged over a large number of different bond configurations, varying from $\mathcal{N}=20 \times 10^{3}$ for the smaller system sizes studied down to $10^{3}$ for the larger ones. For pure Potts models $(r=1)$ error bars follow from averaging over a few hundreds of Monte Carlo runs employing different random numbers, as usual. Finally, for the application of finite-size scaling on the numerical data in terms of characteristic power-law fittings as will be discussed below, we employed the standard criterion of the $\chi^{2} / \mathrm{DOF}$, where DOF denotes the number of degrees of freedom.

\section{FINITE-SIZE SCALING ANALYSIS}

To determine critical properties from Monte Carlo data we use finite-size scaling arguments. For the interfacial adsorption, $W$, one expects [6]

$$
W \approx L^{a} \Omega\left(t L^{1 / \nu}\right)
$$


where the critical exponent $a$ is determined as mentioned above by the bulk critical exponents $\beta$ and $\nu$ via $[6$

$$
a=1-\frac{\beta}{\nu},
$$

$t=\left|T-T_{\mathrm{c}}\right| / T_{\mathrm{c}}$ is the reduced critical temperature and $\Omega$ the scaling function. A more refined ansatz invokes corrections to the asymptotic scaling behavior, as will be discussed below. Note however that in the present work, we are not interested in the temperature dependence of $W$, but rather only on its size dependence. From the above scaling assumption (4) we derive that the leading critical behavior of the interfacial adsorption is given by $W \sim L^{a}[6]$.

At the critical (and tricritical) points, the singularities in the interfacial adsorption are induced by bulk critical fluctuations. On the other hand, at first-order transitions there are no bulk critical fluctuations and the divergence of $W$ arises from an interface delocalisation transition [38]. In the latter case, for lattices of square shapes, a linear divergence of the form $W \sim L$ is expected for the interfacial adsorption at the transition point according to the arguments of Selke et al. [9].

The above scaling predictions have been confirmed reasonably well in previous Monte Carlo simulations for pure $q=3$ Potts and Blume-Capel models showing continuous transitions [6, 8 -10]. On the other hand for the case of first-order transitions where metastability effects cast further difficulties in the scaling analysis, the expected linear divergence has been only partially supported by the numerical data at hand [9, 11]. In the present study, we extend and refine previous results on the size-dependence of the interfacial adsorption by considering the pure Potts model for various values of the internal states $q$ as well as by including bond randomness in the system.

We start our presentation of results by contrasting the critical interfacial adsorption of the pure and disordered 3 -states Potts model in Fig. 2 (the underscore symbols $\mathrm{p}(\mathrm{r})$ in Fig. 2, and following figures as well, refer to the pure(random) cases, respectively). The data for the pure model (filled circles) were taken from Ref. 21] and have been analyzed according to that paper by fitting them (black line in Fig. 2) to equation

$$
W \sim L^{a}\left(1+b L^{-x}\right),
$$

taking into account a possible leading corrections-toscaling exponent $x$. Fixing $x$ to the expected value $4 / 5$ [39 42] the resulting estimate for the critical exponent $a_{\mathrm{p}}=0.870(3)$ agrees within errors with the predicted exact value $a=13 / 15=0.866 \cdots$ (we remind the reader that $\left.(\beta / \nu)_{\mathrm{p}}=2 / 15=0.133 \cdots\right)$ 24]. Accordingly, the findings on the pure 3 -states Potts model strongly support the correctness of the finite-size scaling description (4). However, we point out that the influence of the corrections-to-scaling exponent $x$ is only marginal in these estimations, since, using $x=1$ we find

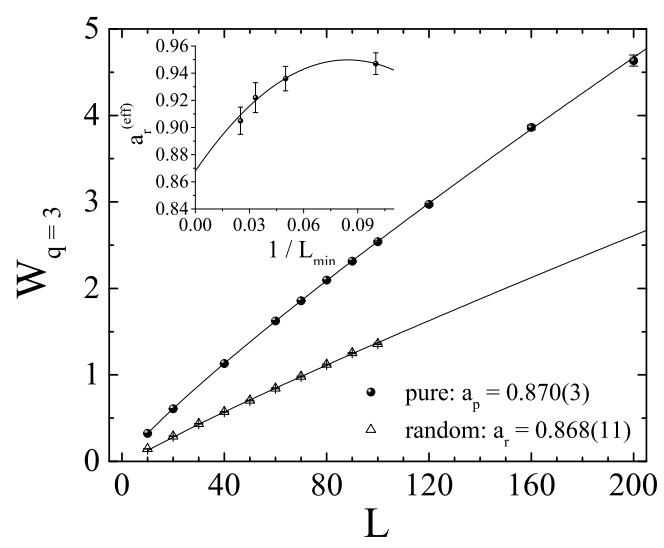

FIG. 2: Finite-size scaling of the interfacial adsorption of the pure and random-bond $q=3$ Potts model. The inset illustrates the infinite limit-size extrapolation of the effective exponent $a_{\mathrm{r}}^{(\mathrm{eff})}$.

$a_{\mathrm{p}}=0.873(4)$ which a bit larger than the expected result, whereas using $x=3 / 5$ we find $a_{\mathrm{p}}=0.867(2)$, which is closer to the exact value.

We continue our presentation with the disordered $q=3$ Potts model. Following previous considerations on its bulk critical properties, we set $r=1 / 10$, where the randomness dominated behavior is expected to show up already for moderate lattice sizes. The arguments leading to this observation were originally discussed by by Wang et al. [43] for the dilute Ising model and later used for other Potts model as well [26, 31 37]. In particular, we monitored, in our simulations, the size dependence of the critical interfacial adsorption. Numerical results for the $q=3$ disordered model are depicted by the open triangles in Fig. 2, Since for the present case and for the subsequent random cases (apart from the $q=4$ case for which logarithmic corrections are known to exist and will be taken into account) there is no clear information with respect to the leading corrections-to-scaling in the literature, and in the light of the above discussion for a marginal effect of the corrections-to-scaling exponent in the fits, we will use the value $x=1$ in Eq. (6) which is the simplest choice leading to reasonable fits. In this way we obtain effective exponents by varying the lower system size $L_{\min }$ included in the fits. A second-order polynomial extrapolation of these effective exponents, as illustrated in the corresponding inset of Fig. 2, provides us with the value $a_{\mathrm{r}}=0.868(11)$. This estimate of $a_{\mathrm{r}}$ is compatible to the value $0.8679(3)$, if one accepts Eq. (5) and the estimate $(\beta / \nu)_{\mathrm{r}}=0.1321(3)$ for the bulk critical exponent ratio of the dilute $q=3$ Potts model given in Ref. [35]. Thus, we may conclude that for the disordered $q=3$ case as well $a_{\mathrm{r}}=1-(\beta / \nu)_{\mathrm{r}}$, in accordance with the finite-size scaling ansatz (4), although it is true that for this particular case there seems to be hardly any difference among the exact value of the exponent ratio $\beta / \nu$ 


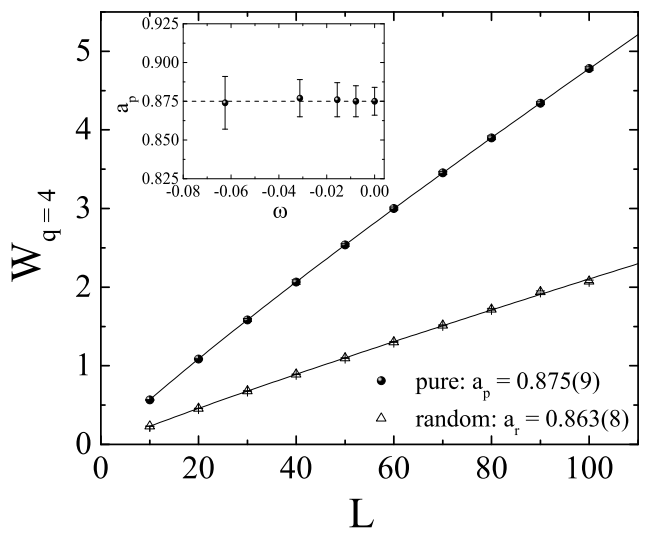

FIG. 3: Finite-size scaling of the interfacial adsorption of the pure and random-bond $q=4$ Potts model. The inset shows fitting results for the exponent $a_{\mathrm{p}}$ of the pure system by varying the value of the corrections exponent $\omega$ within the regime $[-1 / 16,0]$. The dashed line marks the value $a_{\mathrm{p}}=0.875(9)$ that corresponds to the case $\omega=0$.

of the pure model and the corresponding estimates for the disordered model. This fact has also been underlined in an extensive study of the magnetization of the $q=3$ random-bond Potts model by Picco 33 .

Next, we consider the delicate $q=4$ model, for which the size dependence of the interfacial adsorption has never been studied previously. As it is well-known, this is a borderline case of the Potts universality class, where logarithmic corrections are known to exist. In particular, Salas and Sokal [44] have studied in detail the form of these scaling corrections and have found multiplicative logarithmic corrections as well as additive logarithmic corrections, some of which are universal. Of course, numerically observing the existence of logarithmic corrections is always difficult, and it is almost impossible to detect logarithmic corrections using system sizes of the order of $L=100$, as in the present work. However, given the description of these logarithmic corrections in Eqs. (3.21) and (3.22) in Ref. [44], we may try to fit our numerical data using this prescription. Accordingly, a plausible finite-size scaling ansatz for the $q=4$ Potts model has the general form [44]

$$
Q \sim L^{a}[\ln (L)]^{\omega}\left(1+b \frac{\ln [\ln (L)]}{\ln (L)}+b^{\prime} \frac{1}{\ln (L)}\right) .
$$

The corrections exponent $\omega$ takes the value $-1 / 16$ for the absolute magnetization, whereas $\omega=-1 / 8$ for the magnetic susceptibility and $\omega=-3 / 2$ for the specific heat [44 46]. In the light of the previous expectations [6], and given that $W$ scales with an exponent $\beta / \nu$ [see Eq. (5), which also describes the finite-size scaling behavior of the absolute magnetization, one may be tempted to use an exponent $\omega=-1 / 16$. However, the term $[\ln (L)]^{-1 / 16}$ for the system sizes we studied in the present

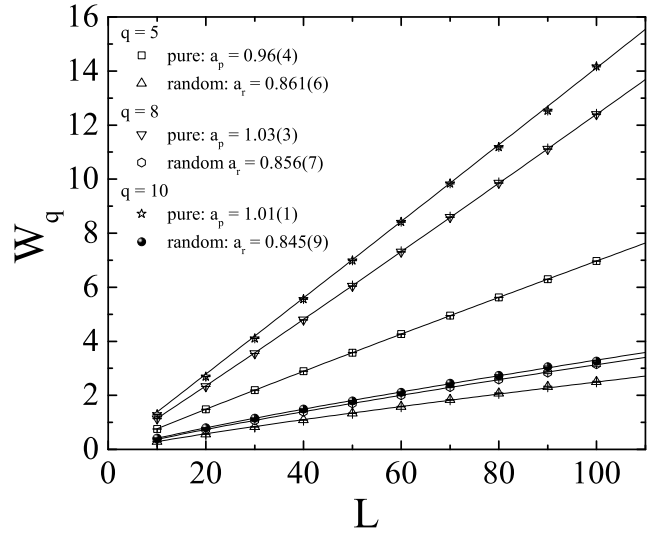

FIG. 4: Finite-size scaling of the interfacial adsorption of the pure and random-bond Potts model for various values of the internal states $q$ in the originally first-order regime, as indicated.

work is close to 1 and we therefore expect that it will have no severe effect in the following fitting attempts. Indeed, we have performed fits of the form (7) on the numerical data of the pure $q=4$ system (shown by filled circles in the main panel of the figure) for several candidate values of $\omega$ within the regime $[-1 / 16,0]$ that verify this expectation. An illustrative plot of our analysis is presented in the inset of Fig. 3 where the estimated values of the exponent $a_{\mathrm{p}}$ are plotted as a function of the fixed exponent $\omega$ used in the fit. The dashed line marks the value $a_{\mathrm{p}}=0.875(9)$ that corresponds to the case $\omega=0$ (illustrated also by the solid black line in the main panel of the figure with an $\chi^{2} / \mathrm{DOF} \approx 0.9$ merit of the fit). Correspondingly, the open triangles in Fig. 3 present our numerical data for the disordered $q=4$ Potts model, for which a fit of the form (7) with $\omega=0$ gives the result $a_{\mathrm{r}}=0.863(8)$, as also indicated in the panel. Again, isotropic scaling and Eq. (5) is satisfied, to a high accuracy for the pure model, for which $(\beta / \nu)_{\mathrm{p}}=1 / 8=0.125$, and within errors for the disordered model for which $(\beta / \nu)_{\mathrm{r}}=0.1385(3)$ [35].

The second part of our study refers to the interfacial properties at the originally first-order transition regime of the Potts model, i.e., for $q>4$. We have simulated the model with internal states $q=5,8$, and $q=10$ in both its pure and disordered $(r=1 / 10)$ version. The data for the disordered $q=8$ case have been taken from Ref. [21]. Our numerical results for the critical interfacial adsorption and the relevant scaling analysis are illustrated in Fig. 4. Several comments are in order: (i) For the pure system we find a clear linear divergence of $W$ for all values of $q>4$ as predicted by scaling arguments $[9]$, since fittings of the form $W \sim L^{a_{\mathrm{p}}}$ give estimates of $a_{\mathrm{p}} \approx 1$ without the need of including scaling corrections. (ii) For the corresponding disordered system fittings of the form (6) with a fixed correction of $x=-1$ give estimates of $a_{\mathrm{r}}$ that 


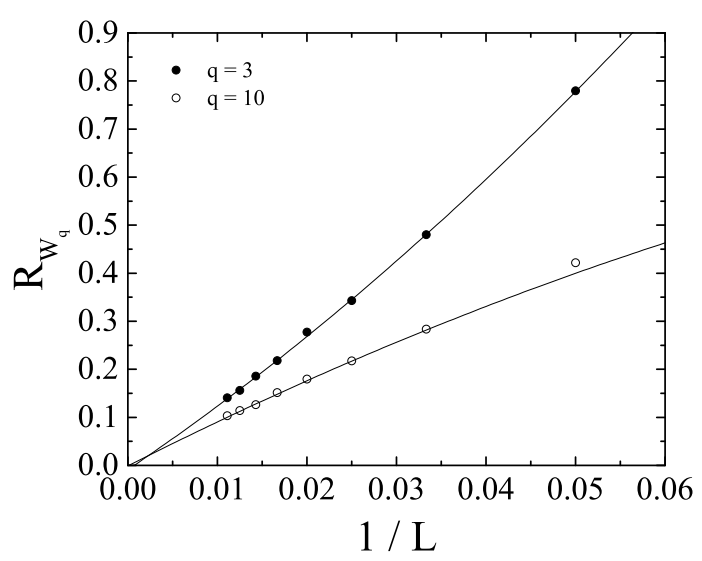

FIG. 5: $R_{\mathrm{W}}$ as a function of the inverse linear system size for two values of $q$, as indicated. The solid lines are second-order polynomial extrapolations to the limit $L \rightarrow \infty$.

again support the isotropic scaling and Eq. (5). Note the most accurate existing estimates of the ratio $(\beta / \nu)_{\mathrm{r}}$ are $0.141(3), 0.145(5)$, and $0.155(5)$ for $q=5,8$, and $q=10$, respectively $32-37]$. (iii) Using a wide range of internal states within the regime $q=3-10$ and two versions of the Potts model, namely the pure model and its disordered counterpart, we have shown that isotropic scaling holds, as well as the relation $a=1-\beta / \nu$.

For all systems studied we also recorded, in addition to the interfacial properties, standard thermodynamic quantities, for both types of boundary conditions. In particular, we measured the specific heat $C_{1: 1}$ and $C_{1: 2}$ and the order parameter given by the majority fraction of the Potts states [24], $m_{1: 1}$ and $m_{1: 2}$. Fittings of $m_{1: 1}$ and $m_{1: 2}$, vanishing at $T_{\mathrm{c}}$ as $\sim L^{-\beta / \nu}$, gave estimates of the magnetic exponent ratio compatible to the above presented results, thus giving further credit to the current numerical data. It is also interesting to note that although the specific-heat data for the pure $q>4$ systems could not be fully described by the standard $L^{2}$ scaling behavior for systems with linear sizes up to $L=100$ (possibly due to strong corrections, especially for the case of the $q=5$ weak first-order transition), we have been able to probe nicely the first-order character of the transition based on the linear divergence of the interfacial adsorption shown for system sizes $L \leq 100$.

As a large part of the current contribution is based on the disordered version of the Potts model, we close this Section with an illustration of the self-averaging properties of the interfacial adsorption. As we know, our numerical studies of disordered systems are carried out using finite samples; each sample is a particular random realization of the quenched disorder. A measurement of a thermodynamic property, say $W$ for the interfacial adsorption considered here, yields a different value for every sample. In an ensemble of disordered samples of linear size $L$ the values of $W$ are distributed according to a probability distribution. The behavior of this distribution is directly related to the issue of self-averaging. In particular, by studying the behavior of the width of this distribution, one may address qualitatively the issue of self-averaging, as has already been stressed by previous authors. In general, we characterize the distribution by its disorder average $[W]$, and also by the relative variance $R_{W}=V_{W} /[W]^{2}$, where $V_{W}=\left[W^{2}\right]-[W]^{2}$. The limiting value of this ratio is indicative of the self-averaging properties of the system [47, 48]. In Fig. [5 we show the infinite limit-size extrapolation of the ratio $R_{W}$ for the random-bond (again $r=1 / 10$ ) Potts model and two selected values of $q$ as indicated in the figure, namely the values $q=3$ (filled circles) and $q=10$ (open circles). For both cases we find that $R_{W} \rightarrow 0$ as $L \rightarrow \infty$, indicating that the interfacial adsorption restores self-averaging in the thermodynamic limit. Similar fitting attempts for other values of $q$, not shown here for brevity, support our conclusion based on these data.

\section{CONCLUSIONS}

We performed extensive Monte Carlo simulations to study the critical interfacial properties in pure and disordered ferromagnetic $q$-states Potts models on the square lattice for various values of the internal states $q \in\{3-$ $10\}$. Interfaces have been introduced by fixing the Potts variables at opposite sites in two different states. The local Metropolis and cluster-flip Wolff algorithms have been used to simulate all systems at their critical points, taking advantage of the existing self-duality. For the disordered models an extensive disorder averaging has been performed in order to control the sample-to-sample fluctuations of the model. The finite-size scaling analysis on our wide-range numerical data allowed us to safely conclude that the isotropic finite-size scaling description for the interfacial adsorption at (pure and randomnessinduced) continuous phase transitions holds. Additionally, for the pure $q>4$ systems that undergo a (weak: $q=5$, or strong: $q=8$ and $q=10$ ) first-order phase transition, we have been able to probe the linear divergence of the interfacial adsorption, verifying the early predictions of the scaling theory. Finally, we have discussed the self-averaging properties of the interfacial adsorption by studying the infinite limit-size extrapolation of properly defined signal-to-noise ratios and we have found that self-averaging is restored in the thermodynamic limit.

\section{Acknowledgments}

The authors would like to thank Prof. W. Selke for suggesting this topic of research, his ongoing collaboration, as well as for many useful discussions and critical comments. We would also like to thank an anonymous Referee for his instructive comments on the problem of corrections-to-scaling in the Potts model. N.G. F. is 
grateful to Coventry University for providing a Research Sabbatical Fellowship during which this work has been completed.

Disorder, and Criticality: Advanced Problems of Phase Transition Theory, Vol. 1, ed. by Y. Holovatch (World Scientific, Singapore, 2004), pp.147-199.

[27] N. Metropolis, A.W. Rosenbluth, M.N. Rosenbluth, M. Teller, and E. Teller, J. Chem. Phys. 21, 1087 (1953).

[28] U. Wolff, Phys. Rev. Lett. 62, 361 (1989).

[29] A. Gamsa and J. Cardy, J. Stat. Mech. (2007) P08020.

[30] D.P. Landau and K. Binder, A Guide to Monte Carlo Simulations in Statistical Physics (Cambridge University Press, Cambridge, 2005).

[31] S. Chen, A.M. Ferrenberg, and D.P. Landau, Phys. Rev. E 52, 1377 (1994).

[32] J. Cardy and J.L. Jacobsen, Phys. Rev. Lett. 79, 4063 (1997).

[33] M. Picco, Phys. Rev. B 54, 14930 (1996).

[34] C. Chatelain and B. Berche, Phys. Rev. Lett 80, 1670 (1997).

[35] C. Chatelain and B. Berche, Phys. Rev. E 60, 3853 (1999).

[36] G. Palagyi, C. Chatelain, B. Berche, and F. Igloi, Eur. Phys. J. B 13, 357 (1999).

[37] J.L. Jacobsen and M. Picco, Phys. Rev. E 61, R13 (2000).

[38] R. Lipowsky, D.M. Kroll, and R.K.P. Zia, Phys. Rev. B 274499 (1983).

[39] Vl.S. Dotsenko and V.A. Fateev, Nucl. Phys. B 240, 312 (1984).

[40] For a review, see V. Privman, P.C. Hohenberg, A. Aharony, in Phase Transitions and Critical Phenomena, ed. by C. Domb and J.L. Lebowitz (Academic Press, New York, 1991), Vol. 14, p.1.

[41] L.N. Shchur, B. Berche, and P. Butera, Phys. Rev. B 77, 144410 (2008).

[42] S.L.A. de Queiroz, J. Phys. A: Math. and Gen. 33, 721 (2000).

[43] J.-S. Wang, V.B. Andreichenko, V.S. Dotsenko, and W. Selke, Physica A 164, 221 (1990).

[44] J. Salas and A.D. Sokal, J. Stat. Phys. 88, 567 (1997).

[45] M. Caselle, R. Tateo, and S. Vinti, Nucl. Phys. B 562, 549 (1999).

[46] L.N. Shchur, B. Berche, and P. Butera, Nucl. Phys. B 811, 491 (2009).

[47] S. Wiseman and E. Domany, Phys. Rev. E 52, 3469 (1995); Phys. Rev. Lett. 81, 22 (1998).

[48] A. Aharony and A.B. Harris, Phys. Rev. Lett. 77, 3700 (1996).

[25] W. Kinzel and E. Domany, Phys. Rev. B 23, 3421 (1981).

[26] For a review, see B. Berche and C. Chatelain, in Order, 